\title{
A influência da atividade física regular sobre o autoconceito
}

\author{
Alvaro Tamayo \\ Ana Paula M. de Campos \\ Daniela R. Matos \\ Graciano R. Mendes \\ Juliana B. dos Santos \\ Nális T. de Carvalho \\ Universidade de Brasília
}

\begin{abstract}
Resumo
Foi objetivo desta pesquisa estudar a influência da atividade física regular de homens e mulheres de mais de 40 anos sobre o seu autoconceito. A Escala Fatorial de Autoconceito foi administrada a 200 sujeitos, metade deles praticando algum tipo de atividade física regular no momento da pesquisa e a outra metade sendo sedentários. A Anova 2X2 revelou efeito principal da atividade física regular e do gênero sobre vários fatores do autoconceito. Os escores foram superiores em autoconfiança, autocontrole e selfsomático para o grupo experimental e no selfético-moral para as mulheres. Conclui-se que a atividade física regular tem impacto benéfico sobre o autoconceito.
\end{abstract}

Palavras-chave: Autoconceito, Atividade física, Gênero.

\begin{abstract}
The influence of regular physical activity on the self-concept. The objective of this research was to study the effect of regular physical activity of men and women over 40 on their self-concept. The Selfconcept Factorial Scale was administered to 200 subjects, half of them practicing some kind of regular physical activity and the other half being sedentary. The Anova 2 X2 revealed a main effect of physical activity and gender on several factors of the self-concept. The scores were higher for the experimental group on self-confidence, self-control, and somatic self. Men scored higher on selfconfidence while women on moral self. It was concluded that regular physical activity is beneficial for self-concept.
\end{abstract}

Key words: Self-concept, Physical activity, Gender

$\mathrm{N}$ este artigo, apresentam-se os resultados de uma pesquisa relativa à influência do gênero e da ativi dade física regular sobre o autoconceito de pessoas adultas, com mais de 40 anos de idade. A relação entre a atividade física e o autoconceito tem sido amplamente estudada com crianças, adolescentes e estudantes universitários, mas as pesquisas com pessoas adultas são raras.

A compreensão do self tem sido uma das metas mais antigas e persistentes da Psicologia (Fiske \& Taylor, 1991). O interesse pelo estudo do autoconceito desenvolveu-se no contexto da fenomenologia existencial e deu origem, rapidamente, a numerosas pesquisas e publicações. O primeiro a analisar sistematicamente a noção de autoconceito foi
William James. A sua contribuição é importante porque constitui uma ruptura com a abordagem filosófica da época e porque introduz a dimensão social no autoconceito. Alguns anos mais tarde, Baldwin apresentou o autoconceito numa perspectiva social e cultural. Esta abordagem social doself foi fortemente reforçada por Cooley (1902) e Mead (1963). Odesenvolvimento do behaviorismo provocou um enfraquecimento do ímpeto dos estudos sobre oself. O estudo de um construto tão "mentalista" como o autoconceito não podia ser estimulado no contexto desta abordagem. Em 1943, Allport advogou pela "re-admissão" do selfna Psicologia. Posteriormente, a crescente influência das abordagens experimental e cognitivista na Psicologia abriu as portas para a 
pesquisa científica do autoconceito e, desde então, o seu estudo não pára de crescer. Segundo Tesser (2000), nos últimos 30 anos o número de pesquisas publicadas triplicou. A década de 50 foi caracterizada principalmente pela preocupação com a mensuração do autoconceito e com o desenvolvimento e validação de numerosos instrumentos de medida (Wylie, 1968; 1974). Paralelamente às pesquisas empíricas, desenvolvera-se a reflexão sobre a natureza doself. Conceitos como protótipo (Rogers, Rogers \& Kuiper, 1979), rede associativa de memória (Bower \& Gilligan, 1979; Greenwald \& Banaji, 1989), esquema cognitivo (Markus, 1977) e autoregulação, isto é, a forma como as pessoas controlam e dirigem as suas próprias ações (Markus \& Wurf, 1987), foram os mais significativos e os que mais têm subsidiado pesquisas empíricas.

O autoconceito pode ser definido como uma estrutura cognitiva que organiza as experiências passadas do indivíduo, reais ou imaginárias, controla o processo informativo relacionado consigo mesmo e exerce uma função de autoregulação (Tamayo, 1993). Niedenthal e Beike (1997) descrevem o autoconceito como as representações mentais das características pessoais utilizadas pelo indivíduo para a definição de si mesmo e regulação do seu comportamento. As representações mentais de que falam os autores têm sido também denominadas esquemas cognitivos ou auto-esquemas. Os esquemas, afirma Cantor (1990), "moldam as percepções que os indivíduos possuem das situações, suas memórias dos eventos e seus sentimentos sobre si mesmos e sobre os outros" (p. 737). Os auto-esquemas resumem as experiências passadas do indivíduo e organizam a ampla variedade de informações relativas a si mesmo (Markus, Crane, Bernstein \& Siladi, 1982). Os múltiplos auto-esquemas de uma pessoa, porém, não podem ser processados simultaneamente; assim, num momento determinado, somente um número limitado deles será processado. Esse conjunto de auto-esquemas que é acessível num determinado momento, constitui o que Markus e Kunda (1986) denominam autoconceito de trabalho. As informações que constituem este último podem ser influenciadas tanto por processos introspectivos internos como pelo contexto externo no qual se encontra a pessoa num momento determinado (Markus \& Wurf, 1987).

Geralmente, consideram-se três componentes no autoconceito: o avaliativo, o cognitivo e o comportamental. O primeiro é denominado auto-estima e consiste na avaliação global que a pessoa faz do seu próprio valor. Normalmente, a auto-estima manifesta-se pela aceitação de si mesmo como pessoa e por sentimentos de valor pessoal e de autoconfiança. Ela constitui um dos determinantes mais importantes do bem-estar psicológico e do funcionamento social (Salmivalli, Kaukiainen, Kaistaniemi \& Lagerspetz,
1999). O componente cognitivo está constituído pelas percepções que o indivíduo tem dos traços, das características e das habilidades que possui ou que pretende possuir. O componente comportamental consiste nas estratégias de autoapresentação utilizadas pelo indivíduo, com o objetivo de transmitir aos outros uma imagem positiva de si mesmo (Schlender, Dlugolecki \& Doherty, 1994). Estes três componentes, avaliativo, cognitivo e comportamental são diferentes, mas estão relacionados entre si. Assim, uma pessoa que se percebe como tendo características indesejáveis, muito provavelmente se avalia de forma desfavorável, mas tenta se apresentar de forma positiva. Tais componentes não são necessariamente estáveis através do tempo e dos múltiplos eventos que ocorrem na vida cotidiana do indivíduo. Recentemente, Nezlek e Plesko (2001) demonstraram que mudanças diárias na clareza do autoconceito covariam com eventos diários positivos e/ou negativos e com mudanças de humor da pessoa. O conteúdo do autoconceito é determinado tanto pela situação social na qual se encontra o sujeito como pelos seus interesses, metas e motivações do momento.

A representação que a pessoa tem de si própria está estocada na sua memória, da mesma forma que a representação que ela tem de outras pessoas ou de objetos. A primeira, porém, é muito mais complexa do que as outras. O selfé uma das estruturas mais elaboradas e mais ricas entre todas as que estão estocadas na memória. Os resultados de numerosas pesquisas revelam que as pessoas memorizam mais facilmente palavras que foram associadas com oself do que com outras pessoas ou com qualquer outra estrutura (Klein, Loftus \& Burton, 1989). Kihlstrom e Cantor (1984) consideram que a representação mental do selfestá constituída por um número indefinido e hierarquizado de autoconceitos específicos, cada um deles representando crenças que a pessoa tem em relação às múltiplas situações da sua existência. Por exemplo, uma pessoa pode ter a percepção de si mesma de que, do ponto de vista social, é tímida, passiva e pouco calorosa. Nas situações de trabalho, a sua percepção pode ser dominada pela eficiência, seriedade e responsabilidade. Apesar de que alguns autores consideram que o selfnão está organizado, a maioria entende que os diversos componentes são estruturas altamente específicas e relacionadas entre si (Higgins, Van Hook \& Dorfman, 1988).

A estrutura cognitiva que constitui o autoconceito controla o processo informativo de duas formas: (1) interpretando, de forma favorável ou desfavorável, as informações relativas ao self e (2) filtrando as informações, de forma a aceitar somente aquelas que são consistentes com a imagem contida no autoconceito. Esta segunda modalidade é exercida através da auto-estima. Campbell e Fairey (1985) e Campbell (1991) mostraram que as pessoas com auto-estima alta processam somente ofeedback positivo e que é consistente com 
o seu autoconceito, ao passo que os indivíduos com autoestima baixa aceitam e são afetados, tanto pelas informações positivas como pelas negativas. Convém lembrar que o autoconceito está constituído por esquemas ou auto-esquemas. Estes funcionam como uma lente que filtra as informações, retendo e integrando somente aquelas que sejam consistentes com o a estrutura dos próprios esquemas.

A função de auto-regulação consiste fundamentalmente em crenças relativas ao controle do comportamento (Thompson, 1981). Por exemplo, crenças sobre habilidades que a pessoa possui para realizar e ter sucesso em determinadas tarefas ou atividades. Numerosas pesquisas têm estudado esta função do autoconceito e na literatura encontra-se a distinção entre controle primário e secundário. O controle primário consiste em tentativas do indivíduo de adaptar o ambiente externo aos próprios desejos e interesses. Compreende controle do comportamento, controle cognitivo, controle da informação e controle da decisão. O controle secundário visa a adaptação do self ao meio ambiente e utiliza mecanismos de controle preditivo, ilusório e interpretativo (Fiske \& Taylor, 1991; Rothbaum, Weisz \& Snyder, 1982). A natureza do autoconceito é dinâmica. Este é um tópico que tem despertado e continua despertando muito interesse da parte dos pesquisadores. $\mathrm{O}$ autoconceito se estrutura através dos vários períodos do desenvolvimento do indivíduo e muda em resposta a modificações no ambiente externo, social e profissional (Kling, Ryff \& Essex, 1997; Nezlek \& Plesko, 2001).

Obviamente, a construção do autoconceito é influenciada pela cultura. O impacto do individualismo e do coletivismo é o que mais tem sido estudado. Grande parte da literatura internacional indica que o indivíduo tem uma forte tendência a manter e promover a sua imagem social através da autoafirmação (Greenwald, 1980; Steele, 1988). Recentemente, pesquisas transculturais, porém, têm revelado que esta auto-afirmação explícita é uma estratégia fundamental de construção e desenvolvimento do autoconceito característica do ocidente, principalmente dos europeus e dos estadunidenses. Esta estratégia parece não ser utilizada em muitos países asiáticos, particularmente no Japão, onde a autocrítica parece ser o elemento fundamental de integração do self (Heine, Takata \& Lehman, 2000; Heine \& Lehman, 1999; John \& Robins, 1994; Kanagawa, Cross \& Markus, 2001; Kitayama \& Karasawa, 1997; Kitayama, Markus, Marsumoto \& Norasakkunkit, 1997). Desta forma, a construção e integração do autoconceito realiza-se através da necessidade de garantir uma percepção positiva dos outros (no ocidente) ou uma percepção positiva de si mesmo (no Japão).

Na multiplicidade de pesquisas publicadas na literatura internacional sobre o autoconceito, este aparece, às vezes, como variável dependente e, às vezes, como independente. São inúmeros os antecedentes, empiricamente observados, do autoconceito. Tanto do ponto de vista teórico como empírico, a fonte mais importante do autoconceito parece ser a forma como a pessoa é percebida pelos outros significativos. A importância dos outros no desenvolvimento e na estabilidade do autoconceito tem sido identificada e salientada explicitamente, não somente pelos pesquisadores, mas também por escritores e filósofos. "Eu reconheço que eu sou como os outros me percebem", afirma Sartre (1943, p. 318). Proust (1954) ratifica esta opinião do filósofo quando afirma: "a nossa personalidade social é criação do pensamento dos outros" (p. 19). Do ponto de vista empírico, a importância dos outros na formação do autoconceito tem sido estudada, no laboratório e em ambiente natural. As pesquisas de laboratório têm-se preocupado principalmente com a mudança provocada no autoconceito em função dofeedback. Os resultados mostram que, em geral, o feedback controlado experimentalmente produz mudanças significativas nas auto-descrições das pessoas. A importância destas mudanças varia em função da favorabilidade dofeedback, da discrepância entre este e o autoconceito e do grau de concordância entre as pessoas que constituem a fonte do feedback (Regan, Gosselink, Hubsch \& Ulsh, 1975; Snyder \& Shenkel, 1976). No ambiente natural, as pesquisas têm focalizado principalmente a relação entre o autoconceito e a maneira como a pessoa é percebida pelos outros significativos (Gray \& Gaier, 1974). Em geral, as pesquisas empíricas nesta área têm seguido direções complementares: o impacto do feedback social sobre o autoconceito (Shrauger \& Lund, 1975), a relação entre o autoconceito e a maneira como o indivíduo é realmente percebido pelos outros (Gray \& Gaier, 1974) e a relação entre o autoconceito e a maneira como o sujeito pensa que é percebido pelos outros (Tamayo, 1985). Segundo Sedikides e Skowronski (1997), o autoconceito se forma e se desenvolve, em grande parte, pela internalização, por parte do indivíduo, da maneira como as pessoas de um grupo o percebem e o avaliam. "What we experience as a self is a reflexive product of social interaction", afirma Cottrell (1969, p. 548). Os outros formam como um espelho no qual, a partir das imagens sociais que ele reflete, o indivíduo se descobre, se estrutura e se reconhece.

O autoconceito é influenciado por muitas outras variáveis de natureza diversa, predominando aquelas com base relacional e social. Algumas das variáveis estudadas têm revelado efeito negativo sobre o autoconceito, tais como neuroticismo, atuação de conflitos nas relações sociais (McClure, Mitchell \& Greschuck, 1982), psicopatia (Tamayo \& Raymond, 1977) e alcoolismo (Gross \& Adler, 1970). Várias características pessoais influenciam significativamente o autoconceito. A título de exemplo podem-se mencionar 
a idade (Kling et al., 1997; L'Ecuyer, 1978), o estado civil (Tamayo, 1986) e a ordem ocupada na família em função do nascimento (Schwab \& Lundgren, 1978; Seff, Gecas \& Frey, 1993). As variáveis que apresentam uma dimensão social mais evidente têm sido freqüentemente estudadas e os resultados observados têm revelado que a influência dessas variáveis é quase sempre positiva para o autoconceito. Por exemplo, a popularidade com os colegas e amigos (Chambliss, Muller, Hulbick \& Wood, 1978), a opinião e feedback do outro significativo (Brennan \& Morris, 1997; Tamayo, 1985), a beleza e aparência física (Aldrige \& Clayton, 1990; Lerner, Orlos \& Knapp, 1976; Mahoney \& Finch, 1976), a frequiência de atividade sexual pré-marital (Tamayo \& Cunha, 1983), e a aceitação social e a atitude positiva dos outros (Tafarodi \& Swann, 1995). O autoconceito é também influenciado por variáveis situacionais, tais como, situação sócio-econômica (Bledson, 1981), posse de carro e índice de acidentes automotores (Tamayo, 1981a), raça e etnia (Osborne \& LeGette, 1982), região e cidade de origem (Paulinelli \& Tamayo, 1987; Tamayo, 1982a) e religiosidade (Blaine, Triverdi \& Ashleman, 1998; Tamayo, 1982b).

A relação do gênero e a identidade sexual com o autoconceito tem sido objeto de numerosas pesquisas. Os resultados ainda não são conclusivos. A revisão da literatura realizada porMacoby e Jacklin (1974) mostrou que os resultados obtidos até o momento eram contraditórios. A ambigüidade persiste em estudos ulteriores. Por exemplo, Stoner e Kaiser (1978) encontraram que em três das 10 escalas do Tennessee Self-Concept Scale os escores eram superiores para os adolescentes masculinos, enquanto que Putnan, Hosie e Hansen (1978), utilizando a mesma escala também com adolescentes, não encontraram nenhuma diferença significativa entre os gêneros. Tamayo (1986), com uma amostra de estudantes universitários, utilizando um instrumento de medida diferente para a avaliação do autoconceito, encontrou que os homens apresentaram escores superiores em autoconfiança e autocontrole, ao passo que as mulheres apresentaram escores superiores no self ético-moral. Outros pesquisadores obtiveram resultados semelhantes (Zuckerman, 1980). Em pesquisa de laboratório e utilizando, como ponto de referência, a rede associativa de memória constituída pelo autoconceito, Josephs, Markus e Tafarodi (1992), encontraram um dos resultados mais original e mais promissor. Os sujeitos da pesquisa foram informados de que eles iam participar numa experiência de construção de frases. A seguir, eles receberam uma lista de 48 palavras com as quais tinham de construir frases. As palavras deviam ser associadas consigo mesmo, com o melhor amigo(a), com o grupo de referência preferido ou com o presidente dos Estados Unidos. Uma vez terminada a etapa de construção de fra- ses, os sujeitos receberam um texto de 20 páginas para ler. Após esta tarefa de interferência, teve um teste surpresa que consistiu em reproduzir, por escrito, o maior número possível das palavras contidas na lista. Os resultados revelaram uma diferença nítida entre homens e mulheres: estas últimas tiveram maior memória de palavras que tinham sido associadas com outras pessoas (melhor amigo ou grupo importante de referência), ao passo que para os homens o maior escore foi em relação a palavras associadas consigo mesmo. Para os autores, este resultado revela uma diferença importante na natureza do conteúdo do autoconceito de homens e mulheres. $\mathrm{O}$ autoconceito masculino estaria mais associado com a própria autonomia e unicidade, enquanto o feminino incluiria, como elemento básico, a referência aos outros significativos.

O impacto do esporte e da atividade física sobre o autoconceito também tem sido abordado em diversas pesquisas. A influência desta variável parece depender, não exclusivamente da ação benéfica da atividade física sobre o funcionamento fisiológico do organismo, mas também da dimensão social presente nesta variável. Várias pesquisas têm mostrado o efeito positivo da atividade física regular sobre a saúde física e mental (Thirlaway \& Benton, 1992). Pesquisas realizadas com crianças, porém, têm dado resultados contraditórios. Hooper, Guthrie e Kelly (1991) observaram que crianças de 8 a 14 anos apresentaram mudanças significativas no autoconceito como conseqüência da sua participação em atividades de treinamento em futebol. $\mathrm{O}$ mesmo efeito foi observado por Hines e Groves (1989) com crianças participando em treinamento de basquete. Smith (1986) também observou o efeito benéfico da participação de crianças de diversos níveis sócio-econômico em atividades esportivas organizadas por centros comunitários. Por outra parte, Leonardson e Garguilo (1978) não observaram relação entre participação de crianças em atividades esportivas e autoconceito e Ames e Ames (1978) encontraram influência negativa. Os resultados contraditórios observados nestas pesquisas podem ser conseqüência de delineamentos de pesquisa inadequados, no sentido de que, no planejamento e na execução da investigação, junto com a variável atividade física ou esportiva, foram incluídas outras variáveis, tais como aprendizagem de novas habilidades esportivas e clima de competição.

Zaharopoulos e Hodge (1992) realizaram um estudo para verificar possíveis diferenças no autoconceito entre atletas e não-atletas. Para este fim utilizaram uma amostra de homens e mulheres entre 13 e 27 anos, sendo que parte deles participava em práticas esportivas e os outros não participavam. Os resultados revelaram que a prática esportiva não tem impacto sobre o autoconceito global mas sobre componentes específicos do mesmo, particularmente o self 
somático. Salokun (1995) estudou, com uma amostra de jovens de 14 a 28 anos, o impacto de progressos realizados nas atividades esportivas e observou correlações positivas entre os ganhos realizados nas habilidades esportivas e vários escores do autoconceito. Delaney e Lee (1996) estudaram a influência da atividade física, competitiva e não-competitiva, com um grupo de adolescentes e jovens de 14 a 27 anos. Os resultados confirmaram o efeito benéfico do exercício físico sobre o autoconceito dos participantes. Kirshton e Dixon (1995) investigaram o impacto da participação, durante cinco semanas, em atividades esportivas organizadas durante o verão para um grupo de jovens dos dois sexos, de nível sócio-econômico inferior, com idade variando entre 10 e 26 anos. O autoconceito foi avaliado no início do programa e no final do mesmo. Os resultados foram favoráveis para os sujeitos de sexo masculino em dois dos seis fatores avaliados. Para as meninas, os escores de autoconceito apresentaram, no final do programa, um certo declínio que os autores interpretaram como sendo conseqüência do estresse provocado pelas competições. Finalmente, Ford, Puckett, Blessing e Tucker (1989) analisaram a influência de oito semanas de participação em um curso de atividades esportivas sobre o autoconceito de estudantes universitários e não observaram nenhuma diferença significativa entre o grupo experimental e o grupo controle. A divergência relativa dos resultados obtidos nas pesquisas relatadas neste parágrafo pode ser devida a diferenças de metodologia e, particularmente, a diferenças na medida do autoconceito bem como a diferenças na natureza das atividades físicas utilizadas como variável dependente.

O estudo do impacto da atividade física sobre o autoconceito tem sido realizado quase exclusivamente com crianças, adolescentes e estudantes universitários e utilizando atividades esportivas, geralmente de curta duração, organizadas por terceiros, realizadas em grupo, freqüentemente relacionadas com a aprendizagem de novas habilidades esportivas e/ou realizadas num contexto de competição. Para o estudo do impacto da atividade física sobre o autoconceito parece relevante, contudo, determinar mais cuidadosamente algumas características da amostra e da própria atividade. Em relação à amostra, não é o mesmo estudar o impacto da atividade física com uma amostra de crianças ou de adolescentes do que com adultos, já estabelecidos familiar e profissionalmente e, em conseqüência, com numerosas obrigações de diversas ordens. Para a criança, para o adolescente e mesmo para o estudante universitário, o esporte, geralmente, faz parte do seu cotidiano. Neste caso, o esporte pode ser procurado e/ou desejado pelo sujeito, mas também ele é, frequientemente, imposto, com horário fixo, infra-estrutura adequada e professor ou instrutor designado. Para o adulto é bem diferente. Ele tem que abrir espaço na sua agenda para praticar uma atividade física e, se esta exigir uma infraestrutura determinada, procurar uma academia, um clube, um parque ou outro local apropriado. Ocasionalmente o adulto pode praticar algum tipo de esporte o atividade física. Mas para estudar o impacto desta sobre o autoconceito, é importante que a atividade não seja simplesmente esporádica, realizada ou organizada somente durante o período de férias, mas que ela apresente regularidade e que não seja prescrita por motivos médicos ou outros.

O objetivo desta pesquisa foi verificar o impacto da atividade física voluntária e regular sobre o autoconceito de pessoas adultas e profissionais. Apesar de considerar que a natureza da atividade física pode ser relevante no estudo da relação desta com o autoconceito, a presente pesquisa teve como alvo estudar a atividade física de forma global, sem considerar a sua natureza. Entende-se aqui por natureza da atividade física o tipo de atividade praticada, por exemplo, esportes bem estruturados com normas e regras estabelecidas e reconhecidas socialmente em oposição a caminhar ou correr, ou ainda, esportes individuais ou coletivos. A variável atividade física foi abordada de forma global, sem discriminar o tipo de atividade nem se ela era praticada individualmente ou em grupo. A atividade, porém, tinha que ser regular, espontânea, não prescrita por motivos de doença ou outros. A falta de estudos empíricos nesta área levou os autores a considerar o estudo da relação entre a atividade física e o autoconceito como sendo uma pesquisa piloto. Desta forma, a variável atividade física regular foi considerada de formaabrangente.

\section{Método}

\section{Amostra}

A amostra foi composta por 200 pessoas de mais de 40 anos, com idade média de 46,12 anos ( $\mathrm{DP}=2,48)$, homens $(57,5 \%)$ e mulheres, sendo que a metade praticava regularmente algum tipo de atividade física e a outra metade não.

\section{Instrumento}

O instrumento utilizado para a avaliação da variável dependente foi uma forma reduzida da EFA: Escala Fatorial de Autoconceito (Tamayo, 1981b), composta por 51 itens com uma escala de 7 pontos. A EFA tem validade fatorial e avalia seis fatores: atitude social, autoconfiança, autocontrole, self ético-moral, self somático e receptividade social. Os coeficientes alpha da versão reduzida são todos superiores a 0,80 . Eles variam de 0,81 a 0,91 . No final da EFA foram colocadas várias perguntas relativas a informações pessoais e, particularmente, à prática regular de alguma atividade física. Duas perguntas referiam-se à atividade física. Na primeira perguntava-se se o sujeito praticava regularmente 
("pelo menos duas vezes por semana") alguma atividade física e, na segunda, se essa atividade era praticada por ordem médica. Os sujeitos que responderam positivamente a esta última questão foram eliminados da amostra.

\section{Procedimento}

Os sujeitos foram abordados pelos pesquisadores que os informavam quanto ao objetivo da pesquisa e o tipo de colaboração solicitado. A EFA foi aplicada em diversos lugares da cidade incluindo parques, clubes, academias e shoppings. Junto com a EFA, os sujeitos recebiam uma prancheta e uma caneta para facilitar a sua tarefa. Para garantir o anonimato, uma vez respondida, a EFA era colocada, pelo próprio sujeito, em um envelope pardo contendo outros questionários já respondidos. Os instrumentos incompletos ou respondidos de forma inadequada foram eliminados.

\section{Resultados e Discussão}

A Anova $2 X 2$ revelou efeito principal da variável gênero sobre os fatores autoconfiança $\mathrm{F}_{(199 ; 1)}=5,71 ; \mathrm{p}<0,01 \mathrm{e}$ self ético-moral $\mathrm{F}_{(199 ; 1)}=6,39 ; \mathrm{p}<0,01$, sendo o escore superior para os homens no primeiro e para as mulheres no segundo. Estes resultados são convergentes com os obtidos em algumas pesquisas anteriores (Tamayo, 1986; Watkins \& Yu, 1993). Como explicar os resultados observados? As diferenças de gênero no autoconceito são necessariamente tributárias das estruturas culturais da sociedade. O fato de ser membro de uma sociedade, de um grupo determinado, cria no indivíduo o sentimento de pertença, define, em grande parte, a sua identidade e, desta forma, influencia significativamente o seu autoconceito. Como foi discutido na introdução, os outros significativos (pais, amigos, professores, esposo/esposa etc.) são determinantes poderosos do autoconceito. Obviamente, esses outros significativos existem num contexto cultural concreto e percebem o mundo e as pessoas a partir de padrões culturais e sociais vigentes, tais como estereótipos sexuais e imagem do homem e da mulher). Os estereótipos sexuais tradicionais e os padrões convencionais de socialização do homem e da mulher poderiam explicar, pelo menos parcialmente, as diferenças de gênero observadas. $\mathrm{O}$ fator de autoconfiança está composto fundamentalmente por itens da categoria de rasgos instrumentais, considerados como característicos do papel sexual masculino (Diaz-Loving, Diaz-Gerrero, Helmreich \& Spence, 1981). Por outra parte, os itens do fator self éticomoral são atributos da categoria de traços expressivos, tais como "bondoso", "generoso", "honesto". Desta forma, as características diferenciais do autoconceito dos homens e das mulheres encontradas nesta pesquisa concordam com o papel instrumental e expressivo, respectivamente. A autoconfiança é uma exigência básica para a ação, para o exercício da atividade profissional, para o sucesso, para construir uma ponte entre a família e a sociedade. No contexto cultural da amostra estudada nesta pesquisa, possivelmente estas eram funções mais características do homem do que da mulher. Em consequiência, ele foi socializado para assumir estas funções. Por outra parte, as mulheres participantes da amostra foram, possivelmente, socializadas com foco nos outros, para sustentar a unidade e a harmonia da família através da consistência moral e ética. Assim, tanto os homens como as mulheres, teriam estruturado o seu autoconceito a partir do espelho social constituído pelos outros significativos, que refletia as suas percepções e atitudes.

A atividade física teve efeito principal sobre os fatores autoconfiança $\mathrm{F}_{(199 ; 1)}=7,57 ; \mathrm{p}<0,006$, autocontrole $\mathrm{F}_{(199 ; 1)}$ $=7,13 ; \mathrm{p}<0,008$, e self somático $\mathrm{F}_{(199 ; 1)}=3,41 ; \mathrm{p}<0,05$, sendo o escore superior para os sujeitos que praticam regularmente algum tipo de atividade física. Foi também observada uma tendência ao nível do self ético moral $\mathrm{F}_{(199 ; 1)}=$ 3,38 ; $\mathrm{p}<0,06$, sendo também o escore superior para os sujeitos que apresentam algum tipo de atividade física. Ao comparar estes resultados com as pesquisas anteriormente discutidas observa-se que, em geral, existe convergência com os resultados nelas obtidos, no sentido do impacto positivo

Tabela 1.

Escore médio em cada um dos fatores da EFA em função da atividade física e do gênero

\begin{tabular}{lcccc}
\hline Fator da EFA & \multicolumn{2}{c}{ Atividade física } & \multicolumn{2}{c}{ Gênero } \\
& Pratica & Não-pratica & Masculino & Feminino \\
\hline Autoconfiança & 5,48 & 5,09 & 5,46 & 5,11 \\
Autocontrole & 5,74 & 5,42 & 5,66 & 5,50 \\
Atitude social & 5,10 & 5,27 & 5,16 & 5,22 \\
Receptividade social & 5,24 & 5,08 & 5,24 & 5,06 \\
Self ético-moral & 6,25 & 6,05 & 6,02 & 6,29 \\
Self somático & 5,21 & 4,98 & 5,13 & 5,04 \\
\hline
\end{tabular}


da atividade física sobre o autoconceito. A comparação, porém, não pode ser direta por tratar-se de pesquisas realizadas com amostras de faixa etária bastante diferente, já que as pesquisas anteriores foram realizadas com crianças, adolescentes e estudantes universitários e esta foi realizada com uma amostra de adultos, profissionais, de mais de 40 anos. Além disso, esta pesquisa difere das outras em três características da atividade física, que era global, regular e voluntária. O efeito observado sobre o self somático não é surpreendente, já que a atividade física, além dos benefícios de tipo estritamente fisiológico, pode apresentar também efeitos positivos de caráter psicológico e social através de modificações, reais ou imaginárias, na estética do corpo que, após os 40 anos, frequientemente, começa a dar origem a diversos tipos de insatisfação. Os padrões culturais atuais insistem na imagem de um corpo atlético, bem proporcionado e com bom condicionamento físico. O simples fato de praticar uma atividade física com regularidade, independentemente dos seus resultados objetivos sobre ofuncionamento e a estética do corpo, pode provocar na pessoa o sentimento, ou a impressão, de que essas exigências normativas da cultura tenham sido, ou estão sendo, atingidas. Desta forma, além dos benefícios estritamente corporais, a atividade física provocaria uma percepção do corpo mais positiva do ponto de vista estético e da saúde.

O efeito observado sobre os fatores autoconfiança, autocontrole e self ético-moral parece, à primeira vista, ser um pouco menos evidente e menos esperado. Contudo, este efeito foi mais significativo do que o impacto da atividade física sobre o self somático. Esta relação pode-se explicar, entre outras coisas, pela dimensão sócio-cultural que apresenta o exercício físico na sociedade atual. Ele é visto como sendo altamente desejável, fonte de saúde física e mental, característico do indivíduo moderno e indicador de assertividade e de autocontrole. Não é surpreendente, portanto, que os que praticam atividade física regularmente, quando comparados com o grupo controle, se percebam com mais autoconfiança, mais autocontrole e, inclusive, mais adaptados às normas éticas e morais da sociedade.

Uma das limitações desta pesquisa foi a falta de controle quanto à natureza da atividade física. Não foi controlado, no levantamento dos dados, se se tratava da prática de algum esporte (tênis, vôlei...), de exercícios realizados em academias, de cooper ou de caminhadas solitárias ou em companhia. Foi simplesmente perguntado se praticavam algum tipo de atividade física. É possível que a prática de esportes estruturados (golfe) versus não estruturados (caminhar) e a prática de esportes individuais (tênis) versus grupais (basquete) influenciem diferencialmente o autoconceito. As pesquisas discutidas na introdução foram realizadas com crianças, adolescentes e estudantes universitários e a variá- vel independente foi, geralmente, um esporte determinado (futebol, basquete...). Não foram encontradas pesquisas comparando o impacto do tipo de esporte sobre o autoconceito. A relevância deste aspecto, contudo, não parece ser muito grande com pessoas da faixa etária utilizada nesta pesquisa.

Outro aspecto que merece ser discutido é o critério de regularidade utilizado nesta pesquisa. Ele foi abrangente e, neste sentido, compreendia desde duas vezes por semana até todos os dias da semana. Como foi discutido anteriormente, a regularidade limitava-se a realizar atividade física, pelo menos, duas vezes por semana. Isto significa que a amostra constituída a partir deste critério pode ter sido relativamente heterogênea do ponto de vista da freqüência da atividade física. A partir dos resultados obtidos, parece indicado utilizar, nas próximas pesquisas, um critério mais rigoroso, considerando como atividade regular aquela que é praticada, por exemplo, pelo menos quatro vezes por semana. Desta forma, poder-se-ia maximizar a relação entre atividade física e autoconceito. Este aspecto parece ser mais relevante para este tipo de pesquisa com adultos do que o relacionado com a natureza da atividade. Sugere-se que em futuras pesquisas com adultos de mais de 40 anos, o critério de regularidade seja mais rigorosamente definido. Inclusive, seria interessante formar subgrupos em função da frequiência da atividade física para verificar se esta tem algum efeito diferencial sobre $\mathrm{o}$ autoconceito.

Finalmente, convém lembrar que sendo esta uma pesquisa de tipo ex post facto as relações observadas entre as variáveis independentes e o autoconceito não podem ser consideradas como expressando uma relação causal. Como neste tipo de estudos as manifestações entre as variáveis independentes e as dependentes já ocorreram no momento em que a pesquisa é realizada, a relação de impacto entre as primeiras e as segundas é meramente inferencial. Assim, as relações observadas nesta pesquisa existem certamente, mas a partir dos resultados obtidos não é possível afirmar nitidamente a causalidade das varáveis independentes sobre a dependente. Apesar desta situação ser comum a todas as pesquisas de tipo expost facto, que constituem a maioria das pesquisas em psicologia, é bom lembrar esta limitação metodológica, a fim de facilitar a interpretação dos resultados obtidos de forma adequada.

\section{Referências}

Aldrige, J., \& Clayton, G. (1990). Sources of self-esteem: perceptions of education majors. College Student Journal, 24, 404-409.

Allport, G. W. (1943). The ego in contemporary psychology. Psychological Review, 50, 451-478.

Ames, C., \& Ames, R. (1978). The thrill of victory and the agony of defeat: Children's self and interpersonal evaluations in competitive and noncompetititve learning environments. Journal of Research and Development Education, 12(1), 79-87. 
Blaine, B. E., Triverdi, P., \& Ashleman, A. (1998). Religious belief and the self-concept: Evaluating the implications for psychological adjustement. Personality and Social Psychology Bulletin, 24(10), 1040-1052.

Bledson, J. (1981). Is self-concept a reliable predictor of economic status? Psychological Reports, 49, 883-886.

Bower, G. H., \& Gilligan, S. G. (1979). Remembering information related to one's self. Journal of Research in Personality, 13, 420-432.

Brennan, K. A., \& Morris, K. A. (1997). Attachment styles, self-esteem, and patterns of feedback seeking from romantic partners. Personality and Social Psychology Bulletin, 23, 23-31.

Campbell, J., \& Fairey, P. J. (1985). Effects of self-steem, hypothetical explanations, and verbalization of expectations on future performance. Journal of Personality and Social Psychology, 48, 1097-1111.

Campbell, J. D. (1991). Self-esteem and clarity of the self-concept. Journal of Personality and Social Psychology, 59(3), 538-549.

Cantor, N. (1990). From thought to behavior: "Having" and "doing" in the study of personality and cognition. American Psychologist, 45, 735-750.

Chambliss, J., Muller, D., Hulbick, R., \& Wood, H. (1978). Relationships between self-concept, self-esteem, popularity and social judgements of junior high school students. The Journal of Psychology, 98, 91-98.

Cooley, D. H. (1902). Human nature and the social order. New York: Scribner's.

Cottrell, L. S. (1969). Interpersonal interaction and the development of the self. In D. A. Goslin (Org.), Handbook of socialization theory and research (pp. 543-570). Chicago: Rand McNally.

Delaney, W., \& Lee, C. (1996). Self-esteem and sex roles among male and female high school students: Their relationship to physical activity. Australian Psychologyst, 30(2), 84-87.

Diaz-Loving, R., Diaz-Guerrero, R., Helmreich, S. T., \& Spence, J. T. (1981). Comparación transcultural y análisis psicométrico de una medida de rasgos masculinos (instrumentales) y femeninos (expresivos). Revista de la Asociación Latinoamericana de Psicología Social, 1, 3-37.

Fiske, S. T., \& Taylor, S., E. (1991). Social cognition (2 ${ }^{\text {nd }}$. ed.). New York: McGraw-Hill.

Ford, H. T., Puckett, J. R., Blessing, D. L., \& Tucker, L. A. (1989). Effects of selected physical activities on health-related fitness and psychological well-being. Psychological Reports, 64(1), 203-208.

Gray, D. F., \& Gaier, E. L. (1974). The congruence of adolescent selfperceptions with those of parents and best friends. Adolescence, 9 299-304.

Greenwald, A. G. (1980). The totalitarian ego: Fabrication and revision of personal history. American Psychologist, 35, 603-618.

Greenwald, A. G., \& Banaji, M. R. (1989). The self as memory system: powerful, but ordinary. Journal of Personality and Social Psychology, 57(1), 41-54

Gross, W. T., \& Adler, L. (1970). Aspects of alcoholics' self concepts as measured by the Tennessee Self-concept Scale. Psychological Reports, 27, 431-434.

Heine, S. J., Takata, T., \& Lehman, D. R. (2000). Beyond self-presentation: Evidence for self-criticism among japanese. Personality and Social Psychology Bulletin, 26(1), 71-78.

Heine, S. J., \& Lehman, D., R. (1999). Cuture, self-discrepancies, and selfsatisfaction. Personality and Social Psychology Bulletin, 25(8), 915-925.

Higgins, E. T., Van Hook, E., \& Dorfman, D. (1988). Does self-attibution form a cognitive structure? Social Cognition, 6 177-207.

Hines, S., \& Groves, D. L. (1989). Sports competition and its influence on self-esteem. Adolescence, 96, 861-869.

Hooper, C., Guthrie, G. D., \& Kelly, T. (1991). Self-concept and skill development in youth soccer players. Perceptual and Motor Skills, $72,275-285$.
John, O. P., \& Robins, R. W. (1994). Accuracy and bias in self-perception: Individual differences in self-enhancement and the role of narcissism. Journal of Personality and Social Psychology, 66, 206-219.

Josephs, R. A., Markus, H. R., \& Tafarodi, R. W. (1992). Gender and selfesteem. Journal of Personality and Social Psychology, 63, 391-402.

Kanagawa, Ch., Cross, S. E., \& Markus, H. R. (2001). "Who am I?" The cultural psychology of the conceptual self. Personality and Social Psychology Bulletin, 27, 90-103.

Kihlstrom, J. F., \& Cantor, N. (1984). Mental representations of the self. In L. Berkowitz (Org.), Advances in experimental social psychology (Vol. 21, pp. 145-180). New York: Academic Press.

Kirshton, J. M., \& Dixon, A., C. (1995). Self-perception changes among sports camp participants. Journal of Social Psychology, 135(2), 135-141.

Kitayama, S., \& Karasawa, M. (1997). Implicit self-esteem in Japan: Name letters and birthday numbers. Personality and Social Psychology Bulletin, 23(7), 736-742.

Kitayama, S., Markus, H. R., Marsumoto, H., \& Norasakkunkit, V. (1997). Individual and collective processus in the construction of the self: self-enhancement in the United States and self-criticism in Japan. Journal of Personality and Social Psychology, 72, 1245-1267.

Klein, S. B., Loftus, J. \& Burton, H. A. (1989). Two self-reference effects: The importance of distinguishing between self-descriptiviness judgements and autobiographical retrieval in self-referent enconding. Journal of Personality and Social Psychology, 56, 853-865.

Kling, K. C., Ryff, C. D., \& Essex, J. M. (1997). Adaptative changes in the self-concept during a life transition. Personality and Social Psychology Bulletin, 23(9), 981-990.

L'Ecuyer, R. (1978). Le concept de soi. Paris: PUF.

Leonardson, G. R., \& Garguilo, R. M. (1978). Self-perception and physical fitness. Perceptual and Motor Skills, 46, 338.

Lerner, R. M., Orlos, J. B., \& Knapp, J. R. (1976). Physical attractiviness, physical effectiveness and self-concept in late adolescents. Adolescence, 11, 313-326.

Macoby, E. A., \& Jacklin, C. N. (1974). The Psychology of sex differences. Stanford: Stanford University Press.

Mahoney, E. K., \& Finch, M. D. (1976). Body-cathexis and self-esteem: a reanalysis of the differential contribution of specific body aspects. Journal of Social Psychology, 99(2), 251-258.

Markus, H. (1977). Self-schemata and processing information about the self. Journal of Personality and Social Psychology, 35, 63-78.

Markus, H., Crane, M., Bernstein, S., \& Siladi, M. (1982). Self-schemas and gender. Journal of Personality and Social Psychology, 42, 38-50.

Markus, H., \& Kunda, Z. (1986). Stability and malleability of the selfconcept. Journal of Personality and Social Psychology, 51, 858-866.

Markus, H., \& Wurf, E. (1987). The dynamic self concept: A social psychological perspective. Annual Review of Psychology, 38, 299-337.

McClure, R., Mitchell, C., \& Greschuck, D. (1982). Self-concept and identification of students needing a counseling center. Psychological Reports, 50, 487-490.

Mead, G. H. (1963). L'esprit, le soi et la société. Paris: PUF.

Nezlek, J. B., \& Plesko, R. M. (2001). Day-to-day relationships among self-concept clarity, self-esteem, daily events, and mood. Personality and Social Psychology Bulletin, 27, 201-211.

Niedenthal, P. M., \& Beike, D. R. (1997). Interrelated and isolated selfconcepts. Personality and Social Psychology Review, 1(2), 106-128.

Osborne, L. W., \& LeGette, H. R. (1982). Sex, race, grade level and social class differences in self-concept. Measurement and Evaluation in Guidance, 14(4), 195-201.

Paulinelli, J. D. C., \& Tamayo, A. (1987). Autoconceito e cidade de origem. Arquivos Brasileiros de Psicologia, 39(1), 68-71. 
Proust, M. (1954). A la recherche du temps perdu (vol. 1). Paris: Bibliothèque NRF de la Pleiade.

Putnan, B. A., Hosie, T. W., \& Hansen, J. L. (1978). Sex differences in self-concept variables and vocacional attitude maturity of adolescents. Journal of Experimental Education, 47, 23-27.

Regan, J. W., Gosselink, H., Hubsch, J., \& Ulsh, E. (1975). Do people have inflated views of their own ability? Journal of Personality and Social Psychology, 31, 295-301.

Rogers, T. B., Rogers, P. J., \& Kuiper, N. A. (1979). Evidence of the self as a cognitive prototype: the "false alarm effect". Personality and Social Psychology Bulletin, 5, 53-56.

Rothbaum, F., Weisz, J. R., \& Snyder, S. S. (1982). Changing the world and changing the self: A two process model of perceived control. Journal of Personality and Social Psychology, 42, 5-37.

Salmivalli, C., Kaukiainen, A., Kaistaniemi, L., \& Lagerspetz, K. M. (1999). Self-evaluated self-esteem, peer-evaluated self-esteem, and defensive egotism as predictors of adolescents' participation in bullying situations. Personality and Social Psychology Bulletin, 25, 1268-1278.

Salokun, S. O. (1995). Positive change in self-concept as a function of improved performance in sports. Perceptual and Motor Skills, 78(3), 752-754.

Sartre, J. P. (1943). L'être et le néant. Paris: Gallimard.

Schlender, B. R., Dlugolecki, D. W., \& Doherty, K. (1994). The impact of self-presentations on self-appraisals and behavior. The power of public commitment. Personality and Social Psychology Bulletin, 20, 20-33.

Schwab, M. R., \& Lundgren, D. C. (1978). Birth order perceived appraisals by significant others, and self-esteem. Psychological Reports, 43, 443-454.

Sedikides, C., \& Skowronski, J. J. (1997). The symbolic self in evolutionary context. Personality and Social Psychology Review, 1(1), 80-102.

Seff, M. A., Gecas, V., \& Frey, J. H. (1993). Birth order, self-concept, and participation in dangerous sports. The Journal of Psychology, 127(2), 231-232.

Shrauger, J. S., \& Lund, A. (1975). Self evaluation and reactions to evaluation from others. Journal of Personality, 43, 94-108.

Smith, T. L. (1986). Self-concepts of youth sport participants and nonparticipants in grades 3 and 6. Perceptual and Motor Skills, 62, 863-866.

Snyder, C. R., \& Shenkel, R. J. (1976). Effects of "favorability" modality, and relevance on acceptance of general personality interpretation prior to and after receiving diagnostic feedback. Journal of Consulting and Clinical Psychology, 44, 34-41.

Steele, C. (1988). The psychology of self-affirmation: Sustaining the integrity of the self. In L. Berkowitz (Org.), Advances in Experimental Social Psychology (Vol. 21, pp. 181-227). San Diego: Academic Press.
Stoner, S., \& Kaiser, L. (1978). Sex differences in self-concept of adolescents. Psychological Repports, 43, 305-306.

Tafarodi, R. W., \& Swann, W. B. J. (1995). Self-liking and self-competence as dimensions of global self-esteem: Initial validation of a measure. Journal of Personality Assessment, 65, 322-342.

Tamayo, A. (1981a). Autoconceito, posse de carro e índice de acidentes automotores. Psicologia: Ciência e Profissão, 1, 101-116.

Tamayo, A. (1981b). Escala Fatorial de Autoconceito. Arquivos Brasileiros de Psicologia, 33(4), 87-102.

Tamayo, A. (1982a). Autoconceito e região de origem. Arquivos Brasileiros de Psicologia, 34(2), 60-64.

Tamayo, A. (1982b). Concept de soi et religion. Psychologica Belgica, 22, $57-65$.

Tamayo, A. (1985). Relação entre o autoconceito e a avaliação percebida de um parceiro significativo. Arquivos Brasileiros de Psicologia, 37(1), 88-96.

Tamayo, A. (1986). Autoconcepto, sexo y estado civil. Acta Psiquiátrica y Psicológica de América Latina, 32, 207-214.

Tamayo, A. (1993). Autoconcepto y prevención. In J. R. Rojas (Org.), Quinta Antologia: Salud y Adolescencia (pp. 18-28). San José de Costarica: Caja Costarricense de Seguro Social.

Tamayo, A., \& Cunha, M. E. (1983). Autoconceito, sexo e frequiência de atividade sexual pré-marital. Ciência e Cultura, 35(7), 800.

Tamayo, A., \& Raymond, F. (1977). Self-concept of psychopaths. The Journal of Psychology, 97, 71-77.

Tesser, A. (2000). On the confluence of self-esteem maintenance mechanisms. Personality and Social Psychology Review, 4 290-299.

Thirlaway, K., \& Benton, D. (1992). Participation in physical activity and cardiovascular fitness have different effects on mental health and mood. Journal of Psychosomatic Research, 36, 657-665.

Thompson, S. C. (1981). Will it hurt less if I can controll it? A complex answer to a simple question. Psychological Bulletin, 90, 89-101.

Watkins, D., \& Yu, J. (1993). Gender differences in the source and level of self-esteem of Chinese college students. Journal of Social Psychology, 133, 347-352.

Wylie, R. C. (1974). The self-concept (Vol. 1). Lincoln: University of Nebraska Press.

Wylie, R. C. (1968). The present status of self theory. In E. F. Borgata \& W. W. Lambert (Orgs.), Handbook of personality theory and research (pp. 728-787). Chicago: Rand McNally.

Zaharopoulos, E., \& Hodge, K. P. (1992). Self-concept and sport participation. New Zealand Journal of Psychology, 20(1), 12-16.

Zuckerman, D. M. (1980). Self-esteem, self-concept, and the life goals and sexrole attitudes of college students. Journal of Personality, 48, 149-162.

Alvaro Tamayo, Doutor em Psicologia Social pela Université de Louvain (Bélgica), é professor do Departamento de Psicologia Social e do Trabalho da Universidade de Brasília (DF). Endereço para correspondência: Alvaro Tamayo, Departamento de Psicologia Social e do Trabalho, Universidade de Brasília, 70.510-900, Brasília, DF.E-mail: tamayo@unb.br.

Ana Paula M. de Campos, Daniela R. Matos, Graciano R. Mendes, Juliana B. dos Santos e Nális T. de Carvalho são estudantes do Curso de Graduação em Psicologia da Universidade de Brasília (DF). 